\title{
Customized, Miniature Rapid-Prototype Stereotactic Frames for Use in Deep Brain Stimulator Surgery: Initial Clinical Methodology and Experience from 263 Patients from 2002 to 2008
}

\author{
Peter E. Konrad ${ }^{a}$ Joseph S. Neimat ${ }^{a}$ Hong Yu ${ }^{c}$ Chris C. Kao ${ }^{a} d$ \\ Michael S. Remple ${ }^{a, d}$ Pierre-François D'Haese ${ }^{b}$ Benoit M. Dawant ${ }^{b}$ \\ Departments of a Neurosurgery and ${ }^{\mathrm{b}}$ Computer Science and Electrical Engineering, Vanderbilt University, \\ Nashville, Tenn., 'Department of Neurosurgery, Stanford University, Stanford, Calif., and d Sentient Medical Systems, \\ Cockeysville, Md., USA
}

\section{Key Words}

Stereotactic surgery $\cdot$ WayPoint $^{\mathrm{TM}} \cdot$ microTargeting $^{\mathrm{TM}}$ platform $\cdot$ Rapid prototyping $\cdot$ Miniature frame $\cdot$ Deep brain stimulators · Parkinson's disease $\cdot$ Essential tremor • Dystonia

\begin{abstract}
Background: The microTargeting ${ }^{\mathrm{TM}}$ platform (MTP) stereotaxy system (FHC Inc., Bowdoin, Me., USA) was FDA approved in 2001 utilizing rapid-prototyping technology to create custom platforms for human stereotaxy procedures. It has also been called the STarFix (surgical targeting fixture) system since it is based on the concept of a patient- and procedurespecific surgical fixture. This is an alternative stereotactic method by which planned trajectories are incorporated into custom-built, miniature stereotactic platforms mounted onto bone fiducial markers. Our goal is to report the clinical experience with this system over a 6-year period. Methods: We present the largest reported series of patients who underwent deep brain stimulation (DBS) implantations using customized rapidly prototyped stereotactic frames (MTP). Clinical experience and technical features for the use of this stereotactic system are described. Final lead location analysis using postoperative $\mathrm{CT}$ was performed to measure the
\end{abstract}

clinical accuracy of the stereotactic system. Results: Our series included 263 patients who underwent 284 DBS implantation surgeries at one institution over a 6-year period. The clinical targeting error without accounting for brain shift in this series was found to be $1.99 \mathrm{~mm}$ (SD 0.9). Operating room time was reduced through earlier incision time by $2 \mathrm{~h}$ per case. Conclusion: Customized, miniature stereotactic frames, namely STarFix platforms, are an acceptable and efficient alternative method for DBS implantation. Its clinical accuracy and outcome are comparable to those associated with traditional stereotactic frame systems.

Copyright $\odot 2010$ S. Karger AG, Basel

\section{Background}

Deep brain stimulation (DBS) is a well-established surgical therapy for movement disorders, and its applications are rapidly expanding into other illnesses such as depression, obsessive compulsive disorder, pain and epilepsy [1-3]. The success of DBS depends on the neurosurgeon's ability to implant electrodes into strategic intracranial targets with extreme precision. Due to the small size and depth of the targets, DBS surgery requires the use of stereotactic methodology to locate intracranial struc-

Peter E. Konrad, MD, PhD

Vanderbilt University Medical Center

Department of Neurosurgery, T-4224 MCN

Nashville, TN 37232 (USA)

Tel. +1 615343 9822, Fax +1 615343 6948, E-Mail peter.konrad@ vanderbilt.edu 
tures in a 3-dimensional space with millimetric accuracy. To achieve this degree of accuracy, DBS surgery has traditionally utilized stereotactic frames, such as the Leksell (Elekta AB, Stockholm, Sweden) or Cosman-RobertsWells (CRW; Integra Radionics, Burlington, Mass., USA), that are mounted to the patient's skull and affixed to the operating table. These frames serve not only as the fiducial system to translate the target coordinates from the patient's image space to their physical space, but also as a rigid fixation method with precise, 3-dimensional positioning that allows the surgeon to place the electrodes at the desired target $[4,5]$. In most DBS implant centers, intraoperative awake physiological mapping is routinely used to verify targeting accuracy and make relatively minor corrections in the final placement of the DBS electrode. The use of traditional stereotactic frames for DBS surgery typically requires the awake patient to undergo reference image acquisition, intraoperative mapping and electrode implantation within the time that the frame is affixed to the patient's head. While this represents the 'gold standard' in stereotactic DBS surgery, several alternative stereotactic methods have arisen to enhance patient comfort, surgical efficiency (namely the amount of continuous time required for the patient to undergo frame application, mapping and DBS electrode placement), and options for bilateral simultaneous mapping and electrode implantation.

An alternative stereotactic method is to create a customized surgical targeting fixture, also referred to as a STarFix (surgical targeting fixture) platform, that can be manufactured with relatively short turnaround (3 days typically) by a rapid-prototyping technology. The senior author (P.K.) began using this method for stereotaxy when first introduced to this system in 2002 as the microTargeting ${ }^{\mathrm{TM}}$ platform (MTP) incorporating STarFix guidance by FHC Inc. (Bowdoin, Me., USA), which was approved for human use by the FDA in 2001. The system includes planning software, bone fiducial markers, and the manufacturing of a customized, miniature stereotactic frame (MTP) that couples with the bone fiducials. Since 2002, we have provided input and tested several iterations of this system over the years. Although previous papers from our institution have documented details regarding the clinical accuracy of the system based on imaging results and virtual testing $[6,7]$, we have yet to report on the general clinical use of this method of stereotaxy for DBS cases. Although a number of clinical centers in the USA and Europe have begun using this method of stereotaxy, there is no paper that describes the initial clinical experience in a large and varied population of DBS

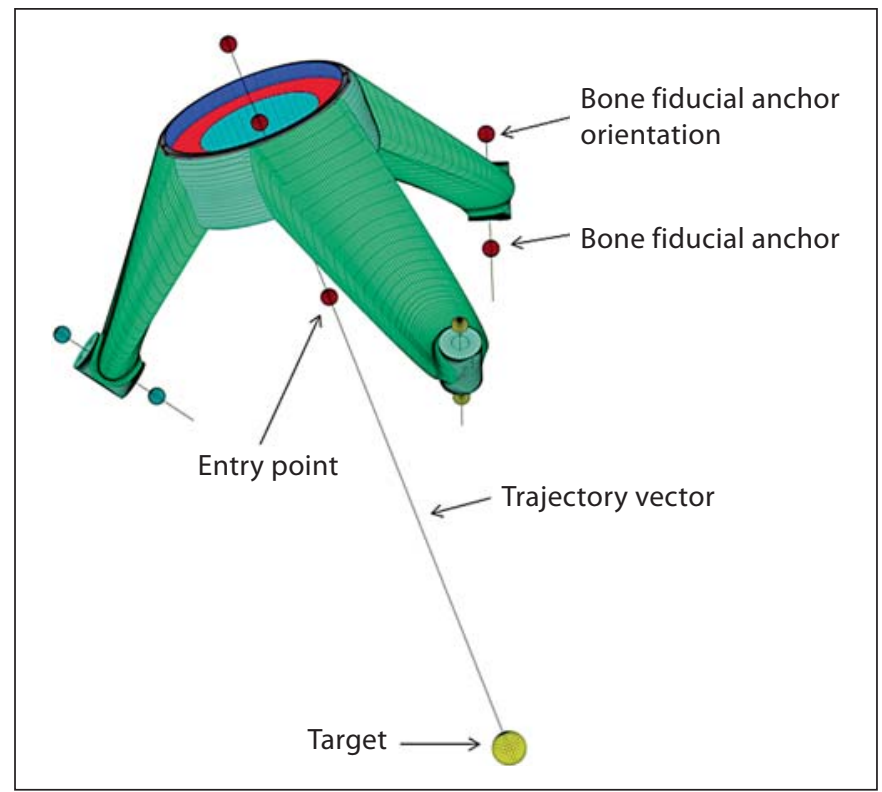

Fig. 1. Virtual computer-aided design diagram of a single, trajectory MTP frame. Four key data points are needed to manufacture an MTP: (1) bone fiducial anchor location; (2) bone fiducial anchor orientation; (3) target; (4) trajectory to target (determined by an entry point selection). In addition, orientation of the trajectory with respect to AC-PC and midline are also included in the computer-aided design file. This assures that the MTP is exactly aligned with patient anatomy, thereby allowing surgeons to easily move the trajectory in parallel or orthogonal directions with respect to the midline. Figure courtesy of Ron Franklin, FHC Inc.

patients using this system. Furthermore, since this method of stereotaxy has rapidly evolved over the past 8 years, there is value in publishing the concepts behind this system and some of the options that rapid prototyping offers surgical targeting. To understand how rapid prototyping of a surgical targeting fixture can be applied to DBS surgery, the following concepts need to be understood.

The STarFix system utilizes bone fiducial markers attached to a customized MTP [8]. Retaining the basic principles of traditional stereotactic frames, the software used to create a rapidly prototyped stereotaxy platform translates the image space to the patient's physical space through skull-mounted fiducials, and target coordinates are referenced to the locations of the same bone fiducial markers (fig. 1). The bone-anchored fiducial markers not only serve as imaging reference points, but also as anchors for a custom-manufactured stereotactic frame. Unlike traditional frames, placement of the fiducial bone anchors occurs in advance of the actual stereotactic frame. Stereotactic planning is performed using the software 

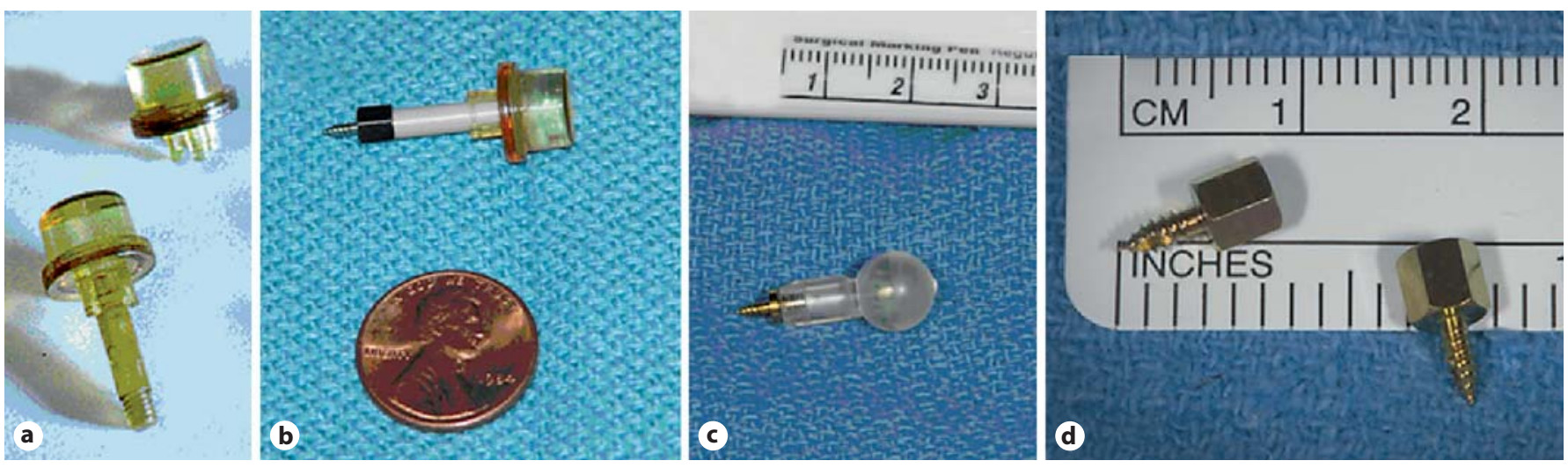

Fig. 2. Evolution of bone fiducial markers used in this report. a Original externalized bone post and imaging cap - Acustar ${ }^{\mathrm{TM}}$ (Z-Kat Inc., Hollywood, Fla., USA) bone post and cap. b Internalized bone anchors with detachable post and imaging cap - WayPoint (FHC Inc.) bone anchors with temporary externalized posts and Acustar cap. c Internalized bone anchors with detachable post and imaging ball - Acustar II. d Internalized anchors only WayPoint ${ }^{\mathrm{TM}}$ bone anchors, which are presently used for all MTP cases at our institution. provided by the manufacturer Voxim $^{\mathrm{TM}}$ or WayPoint ${ }^{\mathrm{TM}}$ planning software, FHC Inc.), and the final computeraided design instruction file is sent in a secure transfer protocol to the manufacturer (FHC Inc.). A custom platform is manufactured using rapid-prototyping technology, and the MTP arrives at the implant center within 3 days of plan submission. Thus, image acquisition must be decoupled from the intraoperative steps, as the MTP is created after the reference images have been obtained and trajectories planned. Each MTP is designed to mate with submillimetric tolerance to the bone fiducial markers, resulting in a rigid but light-weight platform affixed to the skull. Standard microdrives (e.g. microTargeting ${ }^{\mathrm{TM}}$ drive; FHC) used to guide electrodes into the brain are mounted to the MTP. The MTP is custom designed to align the microdrive over the predetermined entry site along the planned trajectory. Once the entire apparatus is assembled, the MTP maintains the trajectories relative to the patient's skull, thereby obviating the need to lock the patient's head to the operating table. The present system is presently marketed under the trade name, WayPoint ${ }^{\mathrm{TM}}$ stereotactic system and sold by FHC Inc.

After initial testing of the STarFix MTP system, the neurosurgeons (P.K. and J.N.) have continued to use it as our first-choice stereotactic system for DBS implantation since 2002. We report our clinical experience from 263 patients who underwent DBS implantation using various iterations of this stereotaxy system at our institution from 2002 to 2008. Analysis of clinical accuracy and operating room usage times were also performed on subsets of pa- tients within this series to allow for comparison of the STarFix system to other available stereotactic systems used for DBS implantation. Data collection and review were approved by the Vanderbilt Institutional Review Board (No. 010809 and 060232).

\section{Methods}

Between 2002 and 2008, 265 patients underwent a total of 288 DBS implantation surgeries at Vanderbilt University Medical Center using the STarFix MTP system as first choice for all DBS surgeries. Surgeries were performed by the first 2 authors (P.K. and J.N.). Under the Vanderbilt Institutional Review Board protocol, all but 2 patients gave consent for their clinical and imaging data to be collected for this study. Thus, surgical complications and clinical outcomes were analyzed for 263 patients and 284 surgeries.

\section{Surgical Technique}

DBS implantation using the MTP was divided into the following 2 stages typically separated by $1-2$ weeks. Each MTP is manufactured from the following data, as illustrated in figure 1: (1) a minimum of 3 fiducial bone anchor locations, (2) orientation of the bone anchors to ensure proper coupling of the MTP legs to the bone anchors, (3) target location with respect to the bone anchors, (4) trajectory to target as determined by an entry point selection. Each platform is manufactured from the 3-dimensional coordinates of the data contained in the patient's CT with fiducials. Furthermore, when identifying the AC-PC and midline structures, the coordinates of these structures are also sent along with the instructional file which assures that each MTP is exactly aligned with the patient's anatomy, thereby allowing parallel or orthogonal trajectory moves with respect to the midline with conceptual simplicity. 
Fig. 3. Single-mount (a) and dual-mount (b) MTPs. The virtual design of these platforms is displayed on the planning software prior to the order submission.
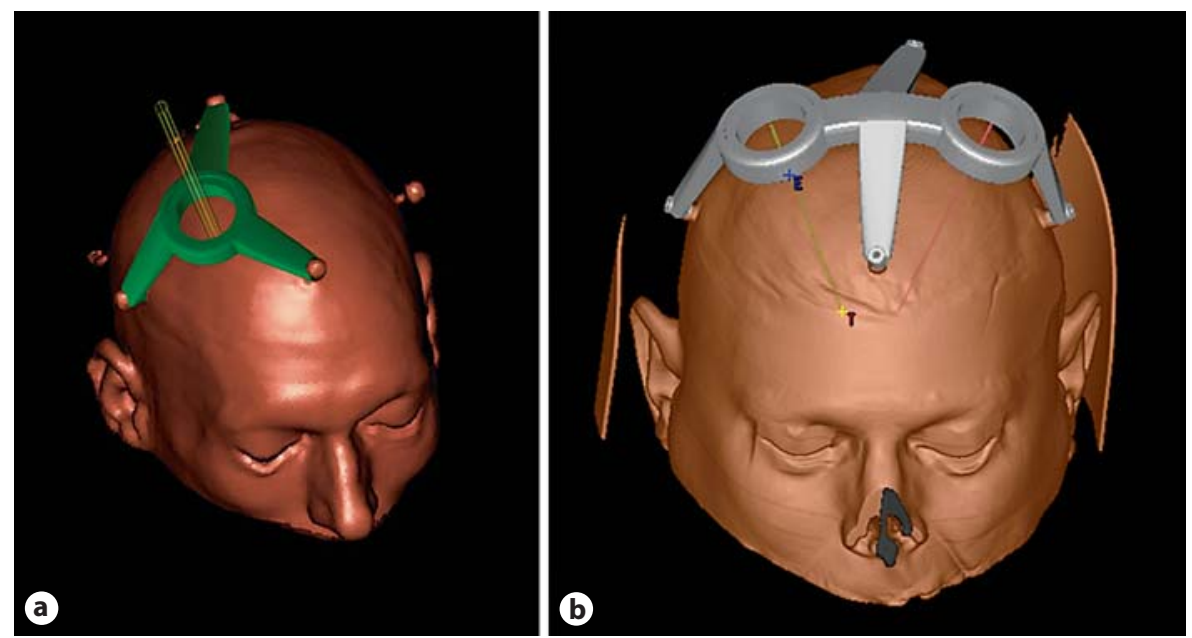

Stage I. Bone fiducial marker implantation and preoperative imaging. The purpose of implanting the bone fiducial markers is twofold: (a) to provide a rigid reference point to allow images to be registered to the patient's physical space and (b) to provide rigid attachment points for a stereotactic frame. The markers must remain fixed to the skull and in the same position from the time of implantation to the time of frame attachment. Figure 2 illustrates the evolution of bone fiducial markers over the course of 6 years included in this report. The earlier generations consisted of imaging caps or balls that snapped onto posts which were attached to anchors screwed into the skull. Posts that protruded through the scalp (fig. 2a) had a propensity for dislodgement in patients with violent movements. We therefore changed to systems with detachable posts or bone anchors that could be completely buried under the scalp. As the planning software improved, external imaging caps or balls were no longer necessary for the coregistration of MRI to CT, and posts were no longer needed to define the orientation of the bone anchors. Since 2005 we have been using the WayPoint ${ }^{\mathrm{TM}}$ (bone anchors (fig. 2d) which are buried completely beneath the scalp, allowing patients to go home without any externalized hardware. Typically, patients were implanted with bone fiducial markers 1 week prior to surgery.

Our technique of WayPoint ${ }^{\mathrm{TM}}$ bone anchor placement is described as follows. We prefer to acquire both the CT and MRI under general anesthesia to ensure motion-free images, which we feel improves the accuracy of trajectory planning. We coordinated the bone anchor placement with the image acquisition as one outpatient procedure. Once the patient was anesthetized on the CT table, the scalp was shaved and prepared via standard sterile technique. Each bone anchor was screwed into the outer table of the skull through a small stab incision in the scalp. The stab incisions were then closed with staples. Three bone anchors were placed in patients undergoing unilateral DBS implantation, while 4-6 bone anchors were placed in those undergoing bilateral DBS implantation. Noncontrasted CT images were acquired with $512 \times 512$ pixels and $0.5-1-\mathrm{mm}$ slice thickness. Contrast-enhanced MRI volumes were obtained on a 1.5- or 3-tesla magnet (3-dimensional SPGR volumes, TR: 12.2, TE: 2.4; $256 \times 256 \times$ 170 voxels; typical voxel dimension of $1 \times 1 \times 1 \mathrm{~mm})$. No anti- biotics were administered either during bone anchor placement or following the procedure. Patients were sent home with instructions to keep the bone anchor sites clean by applying hydrogen peroxide to the anchor sites twice a day until the day of surgery.

Several commercially available software planning programs are compatible with the STarFix MTP system. During the course of this series, we have used the Voxim ${ }^{\mathrm{TM}}$ and microTargeting ${ }^{\mathrm{TM}}$ WayPoint ${ }^{\mathrm{TM}}$ planner (available through FHC Inc.), and also the StimPilot ${ }^{\mathrm{TM}}$ (available through Medtronic Neurological Inc., Minneapolis, Minn., USA). In addition, we have begun developing an MTP planning software (CRAVE) that is integrated with a deformable physiological atlas [9-11]. With any of the software mentioned above, the digital CT and MRI Digital Imaging and Communications in Medicine files were imported into the planning software and coregistered. Targets were selected on the MRI images using the Schaltenbrand and Wahren Atlas coordinates for target nuclei [12-16]. Entry points were selected to avoid crossing vascular structures. Bone fiducial markers were identified on the CT images. Once the target, trajectory and bone fiducial markers were identified, a customized MTP was designed by the software. As shown in figure 3, either single- or dual-trajectory MTP frames can be ordered. Once the MTP design was verified, the file containing the specifications for manufacturing an MTP was submitted to the manufacturer by a secure internet file transfer protocol. The trajectory-specific MTP arrived at our hospital within 3 days.

Stage II. Intraoperative mapping and electrode implantation. Most patients underwent this stage awake with local anesthetics. General anesthesia was reserved for patients with violent movements from generalized dystonia. The patient's head was supported by a padded headrest but not locked to the operating table. The MTP was rigidly connected to the existing bone anchors using couplers provided by the manufacturer (fig. 4). The entry point was marked on the scalp and skull using a guide that fits through the ring opening of the MTP. A 14-mm diameter burr hole was then drilled at the planned entry point. An electronic microTargeting $^{\mathrm{TM}}$ drive (FHC) was mounted onto the MTP and guided electrodes along the planned trajectories for mapping. Standard physiological mapping using microelectrode recording (MER) and/or macrostimulation techniques were performed [13, 16-18]. 
MER responses were measured at $0.5-\mathrm{mm}$ increments along each trajectory, typically starting at $10 \mathrm{~mm}$ above and ending at $5 \mathrm{~mm}$ below the planned target. Macrostimulation responses were recorded at 1- to 2-mm increments. The depth of the electrode tip with respect to the planned target was displayed in the software and was correlated with the drive setting. If necessary, alternate tracks were tested by passing cannulas through parallel guide tubes in the drive assembly. Various offset adapters for the drive assembly allowed target and entry adjustments within a $22-\mathrm{mm}$ diameter centered on the planned trajectory. The exact coordinates for each tract were displayed on the microTargeting ${ }^{\mathrm{TM}}$ WayPoint ${ }^{\mathrm{TM}}$ planner, which also contained the option to display all possible tracks on the preoperative MRI.

Once the optimal tract and target were identified by physiological mapping, the test electrode was replaced by the quadripolar DBS lead (model 3387 or 3389; Medtronic Neurological Inc.). Fluoroscopy was initially used during the first 2 years to observe the exchange of the MER or semimicrostimulating electrodes with the quadripolar DBS lead. However, since the patient's head was not affixed to the operating table and the STarFix MTP system did not include a radiographic reference guide, manual orthogonal alignment of the patient's head in the fluoroscopic X-ray beam was difficult and eventually abandoned. Since 2004 we have not used fluoroscopy to verify the final exchange of electrodes. Instead, we rely on physiological testing of the implanted DBS lead to confirm proper targeting. This is done by the neurologist who would typically perform the postoperative programming of the device.

The final DBS lead was anchored to the skull using either the Stimloc ${ }^{\mathrm{TM}}$ device (Medtronic Neurological Inc.) or methylmethacrylate, and the extracranial end of the lead was buried beneath the scalp. The MTP and bone fiducial markers were removed at the end of the surgery. A postoperative CT was obtained for all patients within $24 \mathrm{~h}$ to identify any structural complications.

Final DBS electrode connection to an internal pulse generator was usually performed as an outpatient procedure under general anesthesia on an alternate day.

\section{Targeting Error Analysis}

For patients who underwent the appropriate postoperative CT protocol (i.e. $512 \times 512$ pixels with $0.5-1-\mathrm{mm}$ slice thickness), we used a previously described lead extraction algorithm to identify the coordinates of the actual implant target [19]. The method we used to calculate the targeting error was previously published in D'Haese et al. [19] and briefly summarized here. Once the optimal target was determined based on intraoperative mapping, we used the planning software to calculate the position of the midpoint between the 2 middle contacts, which we defined as the calculated implant target, in preoperative CT coordinates. An automatic algorithm detected the DBS lead artifact on the postoperative CT and reported the coordinate of the midpoint between the 2 middle contacts, which was defined as the actual implant target coordinate. By fusing the preoperative CT to the postoperative CT for each patient, the calculated implant target coordinate and the actual implant target coordinate were translated into the same reference space. The distance between the actual and calculated implant target coordinates was defined as the targeting error. This targeting error includes not only the software and frame manufacturing error, but also the surgical placement error and brain shift error. This was computed for 75 patients in this series.

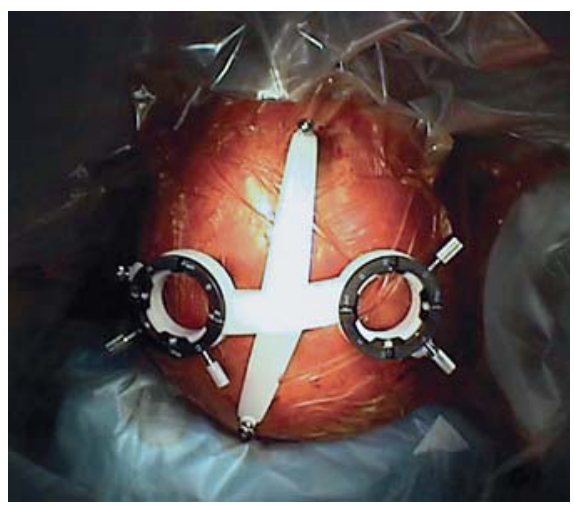

Fig. 4. Example of a bilateral MTP mounted on a patient prior to mounting the microdrives.

\section{Time Usage Analysis}

Total surgical time and incision start times were collected for 10 random patients within this series who underwent bilateral DBS implantation by the senior author (P.K.). Analogous data was collected for 10 patients who underwent DBS implantation using the CRW frame by the same surgeon. The CRW cases were from 2001, since we changed to the STarFix system starting in 2002. Patients were matched based on disease.

\section{Results}

The 263 patients included in this series underwent 284 DBS implantation surgeries. A total of 497 leads were implanted in these patients. The indications for DBS were the following: Parkinson's disease $(\mathrm{n}=139)$, essential tremor $(n=89)$, generalized dystonia $(n=31)$, central neuropathic pain $(n=3)$ and failure of previously implanted DBS lead $(n=1)$.

We encountered the following technical complications. In 3 patients $(0.1 \%)$ with severe dyskinesias, one or more bone fiducial markers (Acustar ${ }^{\mathrm{TM}}$ early version) were dislodged before stage II. These patients later underwent DBS implantation using the CRW system. After the switch to the detachable posts and ultimately to the bone anchor only system (fig. 2), there were no further incidents of broken markers. One patient $(0.004 \%)$ had a bone fiducial marker infection that resolved after marker removal and antibiotic therapy. After the infection had cleared, that patient underwent DBS implantation using the CRW frame. In another patient (0.004\%), the MTP did not fit onto all the bone anchors due to anchor localization error during planning. For that patient, surgery was postponed until a new MTP was created, and 
subsequent DBS implantation proceeded without problems.

The targeting error of the MTP system was measured in 75 patients for whom appropriate postoperative CT was available for analysis. We found this error to be an average of $1.99 \mathrm{~mm}$ (SD 0.9), and it represents the vector distance from the intended midpoint of the DBS electrode to the measured midpoint of the implanted DBS electrode on the immediate postoperative CT scan. Since the method of error calculation described above does not account for brain shift or error introduced during electrode fixation, the actual targeting error of the STarFix system is less than $1.99 \mathrm{~mm}$. A recent study published by our group further separated this series of patients into subgroups based on the amount of brain shift. When brain shift was minimal, targeting error was reduced to a mean of $1.24 \mathrm{~mm}$ (SD 0.4) [19].

Based on our operating room usage analysis, we found that the total operating room time did not differ significantly between CRW versus STarFix cases (309 min vs. 280 min). However, incision start time in STarFix cases was on average $173 \mathrm{~min}(\mathrm{p}<0.01)$ earlier than in comparable CRW cases. This translated to shorter operating room usage times per case and allows us to perform 2 DBS implantation surgeries on the same day.

\section{Discussion}

This report represents our methodology and experience with the initial use of the STarFix MTP system for patients undergoing DBS implantation. It is the largest experience reported to date for the use of this system. This report also documents, for the first time, the methodology of using a rapidly prototyped stereotactic frame as it evolved from the initial experience in 2002 until 2008. We also compared the operative times from our experience with the STarFix system to the CRW stereotactic system used previously.

Our incidence of technical complications using the STarFix MTP system was quite low ( $0.1 \%$ or less). The cases of bone marker dislodgement are conceptually similar to cases where the traditional stereotactic frame becomes detached from the skull, thereby requiring replacement of the frame. With refinement of bone fiducial marker design, this problem has become extremely rare. The case in which the platform failed to mount correctly illustrates the importance of accurate fiducial registration during planning, a fact that also applies to traditional stereotactic frames.

Rapid-Prototype Stereotaxy Frames for DBS Surgery
Due to the precision required for DBS implantation, much emphasis has been placed on the accuracy of various stereotactic systems. Accuracy is inversely related to the targeting error, which is the distance between the actual implant coordinate and the calculated implant coordinate from the stereotactic system. In phantom models, the Leksell and CRW frames have published targeting errors of $1.7 \mathrm{~mm}$ (SD 1.0) and $1.8 \mathrm{~mm}$ (SD 1.1), respectively [20]. The targeting error of STarFix MTPs as studied in phantom models is $0.42 \mathrm{~mm}$ (SD 0.15) [6]. The Nexframe $^{\mathrm{TM}}$ (Medtronic Neurological Inc.), which is a frameless stereotactic system, has a targeting error of $1.25 \mathrm{~mm}$ (SD 0.6) in phantom models [21]. When trying to compare clinical accuracy of various frame systems, the error is confounded by brain shift during the procedure and from lead fixation. Thus, most measured targeting errors in clinical settings are overestimations of the actual targeting error of the stereotactic system. Recently, Kelman et al. [22] measured the targeting errors from a large series of patients who underwent DBS implantation using the Nexframe ${ }^{\mathrm{TM}}$ or CRW frame, and found targeting errors of $2.78 \mathrm{~mm}$ (SD 0.25) for the Nexframe ${ }^{\mathrm{TM}}$ and 2.65 $\mathrm{mm}$ (SD 0.22) for the CRW frame. We found a targeting error of $1.99 \mathrm{~mm}$ (SD 0.92) for the MTP system in the clinical setting. This suggests that the STarFix MTP system is at least as accurate as these other systems.

We observed several advantages in using a customized, miniature frame over a traditional stereotactic frame. Eliminating the bulk of the frame and the need to secure the patient's head to the operating table allowed patients to adjust their head position during intraoperative mapping, which helped to overcome anxiety and reduce discomfort. Effects of test stimulation on head and neck tremor or dystonia can be more easily detected intraoperatively when the head is not locked to the table.

Since January 2005, bilateral implantations have been performed with dual-mount MTPs (fig. 4).

The dual-mount MTP allows simultaneous bilateral MER with the use of 2 drives and a multichannel recording system. The ability to record MER data from bilateral nuclei provides a unique scientific opportunity to compare physiology in homologous nuclei while exposing the patient to various tasking or stimulation paradigms. By our estimations, it has also reduced the MER recording time by nearly half at our institution.

By placing the fiducial anchors and acquiring the images (stage I) on a separate day from the stereotactic mapping and lead placement (stage II), several advantages became apparent. Motion-free images can be obtained on a day prior to surgery when the patient can be fully anes-

Stereotact Funct Neurosurg 2011;89:34-41 
thetized without compromising intraoperative physiological mapping. For most traditional frame cases, the need to mount the frame and obtain at least a preoperative $\mathrm{CT}$ on the same day as intraoperative mapping inevitably delays incision time. In our experience, incision start times for the STarFix cases were typically $2 \mathrm{~h}$ earlier than CRW cases. This translated to shorter operating room usage times. Since previous studies have shown that fiducial bone markers can remain implanted for over 3 weeks without added risk of infection [23, 24], the same MTP and bone anchors can be used for multiple procedures without reapplying new bone markers or ordering another MTP frame. This provides the option of staging bilateral DBS implantations without having to repeat the planning process.

Most of the advantages of miniature stereotactic frames described above can also be found in the Nexframe ${ }^{\mathrm{TM}}$ system, which utilizes bone fiducial markers to relate the image space to the patient's physical space and uses a light-weight skull-mounted tower to help guide the electrodes without rigidly fixing the head to the table. The fundamental difference between a custom, rapidly prototyped frame such as MTP and the Nexframe ${ }^{\mathrm{TM}}$ lies in the trajectory alignment method. The STarFix MTP system incorporates traditional stereotactic frame principles of translating the trajectory coordinates mathematically into the platform by coupling with bone fiducials (i.e. the registration and trajectory alignment is built into the MTP as seen in fig. 1). Nexframe ${ }^{\mathrm{TM}}$, on the other hand, requires optical tracking devices to manually register and align the trajectory during surgery $[25,26]$. Precise intraoperative registration and alignment are critical to ensure the accuracy of the Nexframe ${ }^{\mathrm{TM}}$ system. Thus, conceptually, STarFix platforms are much closer in design concept to the traditional stereotactic frames.

Disadvantages of a rapidly prototyped stereotaxy system include the following. Due to the need for frame manufacturing and shipping, stage I and II of the procedure must be spaced by at least 3 days in the case of the MTP system from FHC. This has not been a problem for our elective DBS practice, and patients have accepted this well within our own practice. Any error in MTP manufacturing could theoretically cause failure of proper frame mounting or a misplaced electrode. The quality control inherent in the rapid prototyping process has been extremely stringent, and we have not encountered an MTP manufacturing error during the entire period of this study. Our 1 case of MTP mounting failure was not due to a manufacturing error but to a fiducial registration error during the planning step. Implanted bone fiducial markers carry a small risk of infection; in our series that risk was $0.004 \%$. Finally, intraoperative adjustment of trajectories is presently limited to a $22-\mathrm{mm}$ diameter area, with any movement outside of that area necessitating a new MTP to be generated. With appropriate preoperative trajectory planning, we have not found the need to map beyond the $22-\mathrm{mm}$ diameter restriction.

\section{Conclusion}

This report provides the first clinical methodology of a customized, miniature, rapidly prototyped stereotactic system (presently known as the WayPoint ${ }^{\mathrm{TM}}$ microTargeting $^{\mathrm{TM}}$ stereotaxy system; FHC Inc.) as an initial user site from the years 2002-2008. Our extensive clinical experience indicates that use of the rapidly prototyped MTP system is an accurate, safe and efficient alternative stereotactic system for the implantation of DBS electrodes. We have been pleased with its simplicity of use and improved patient comfort for elective DBS surgeries.

\section{Acknowledgements}

The authors wish to acknowledge the help and support of Melba Isom and Gilbert Humbert (Vanderbilt University) and Ron Franklin (FHC Inc.) in helping with the submission of the manuscript.

\section{Disclosure Statement}

This work was partially supported by funds from the $\mathrm{Na}$ tional Institutes of Health grant (NIH-NIBIB, 1 R01-EB006136). B.M.D. receives a portion of the royalties paid by FHC, Inc. to Vanderbilt for the license of the WayPoint ${ }^{\mathrm{TM}}$ Planner used to plan some of the procedures.

References

Konrad/Neimat/Yu/Kao/Remple/ D'Haese/Dawant 
4 Galloway RL, Maciunas RJ: Stereotactic neurosurgery. Crit Rev Biomed Eng 1990;18: 181-205.

5 Galloway RL Jr, Maciunas RJ, Latimer JW: The accuracies of four stereotactic frame systems: an independent assessment. Biomed Instrum Technol 1991;25:457-460.

-6 Balachandran R, Mitchell JE, Dawant BM Fitzpatrick JM: Accuracy evaluation of microtargeting platforms for deep-brain stimulation using virtual targets. IEEE Trans Biomed Eng 2009;56:37-44.

7 Fitzpatrick JM, Konrad PE, Nickele C, Cetinkaya E, Kao C: Accuracy of customized miniature stereotactic platforms. Stereotact Funct Neurosurg 2005;83:25-31.

8 FHC Corporation: Starfix microtargeting platform patent. USA, 2001.

$\checkmark 9$ D’Haese PF, Cetinkaya E, Konrad PE, Kao C, Dawant BM: Computer-aided placement of deep brain stimulators: From planning to intraoperative guidance. IEEE Trans Med Imaging 2005;24:1469-1478.

- 10 D'Haese PF, Pallavaram S, Niermann K, Spooner J, Kao C, Konrad PE, Dawant BM: Automatic selection of DBS target points using multiple electrophysiological atlases. Med Image Comput Comput Assist Interv 2005;8:427-434.

11 Pallavaram S, D'Haese PF, Kao C, Yu H, Remple M, Neimat J, Konrad P, Dawant B: A new method for creating electrophysiological maps for DBS surgery and their application to surgical guidance. Med Image Comput Comput Assist Interv 2008;11:670-677.

-12 Hutchison WD, Allan RJ, Opitz H, Levy R, Dostrovsky JO, Lang AE, Lozano AM: Neurophysiological identification of the subthalamic nucleus in surgery for Parkinson's disease. Ann Neurol 1998;44:622-628.
13 Starr PA, Christine CW, Theodosopoulos PV, Lindsey N, Byrd D, Mosley A, Marks WJ Jr: Implantation of deep brain stimulators into the subthalamic nucleus: technical approach and magnetic resonance imagingverified lead locations. J Neurosurg 2002;97: 370-387.

- 14 Tan AK, Yeo TT, Tjia HT, Khanna S, Nowinski WL: Stereotactic microelectrode-guided posteroventral pallidotomy and pallidal deep brain stimulation for Parkinson's disease. Ann Acad Med Singapore 1998;27:767771.

15 Volkmann J, Benecke R: Deep brain stimulation for dystonia: patient selection and evaluation. Mov Disord 2002;17(suppl 3):S112S115.

16 Zonenshayn M, Rezai AR, Mogilner AY, Beric A, Sterio D, Kelly PJ: Comparison of anatomic and neurophysiological methods for subthalamic nucleus targeting. Neurosurgery 2000;47:282-292, discussion 292294.

17 Pralong E, Ghika J, Temperli P, Pollo C, Vingerhoets F, Villemure JG: Electrophysiological localization of the subthalamic nucleus in parkinsonian patients. Neurosci Lett 2002;325:144-146.

18 Gill CE, Konrad PE, Davis TL, Charles D: Deep brain stimulation for Parkinson's disease: the Vanderbilt University Medical Center experience, 1998-2004. Tenn Med 2007; 100:45-47
19 D’Haese PF, Pallavaram S, Konrad PE, Neimat J, Fitzpatrick JM, Dawant BM: Clinical accuracy of a customized stereotactic platform for deep brain stimulation after accounting for brain shift. Stereotact Funct Neurosurg 2010;88:81-87.

20 Maciunas RJ, Galloway RL Jr, Latimer JW: The application accuracy of stereotactic frames. Neurosurgery 1994;35:682-694, discussion 694-695.

-21 Henderson JM, Holloway KL, Gaede SE, Rosenow JM: The application accuracy of a skull-mounted trajectory guide system for image-guided functional neurosurgery. Comput Aided Surg 2004;9:155-160.

-22 Kelman C, Ramakrishnan V, Davies A, Holloway K: Analysis of stereotactic accuracy of the Cosman-Robert-Wells frame and Nexframe frameless systems in deep brain stimulation surgery. Stereotact Funct Neurosurg 2010;88:288-295.

23 Vinas FC, Zamorano L, Buciuc R, Li QH, Shamsa F, Jiang Z, Diaz FG: Application accuracy study of a semipermanent fiducial system for frameless stereotaxis. Comput Aided Surg 1997;2:257-263.

>24 Maurer CR Jr, Fitzpatrick JM, Wang MY, Galloway RL Jr, Maciunas RJ, Allen GS: Registration of head volume images using implantable fiducial markers. IEEE Trans Med Imaging 1997;16:447-462.

25 Henderson JM: Frameless localization for functional neurosurgical procedures: a preliminary accuracy study. Stereotact Funct Neurosurg 2004;82:135-141.

-26 Holloway KL, Gaede SE, Starr PA, Rosenow JM, Ramakrishnan V, Henderson JM: Frameless stereotaxy using bone fiducial markers for deep brain stimulation. J Neurosurg 2005;103:404-413. 